\title{
Application and research on on-line automatic-monitoring platform of radiation sources based on the data communication optimization
}

\author{
Dongli Yang \\ School of computer \& information technology \\ Northeast Petroleum University \\ Daqing, HeiLongjiang ,China \\ yangdl_2003@163.com
}

\author{
Bin Yang \\ Technical Physics Institute of Heilongjiang Academy of \\ science \\ Harbin, HeiLongjiang,China \\ johnyangbin@163.com
}

\begin{abstract}
This thesis summarized the content of IOCP( I/O completion port ). It was improved in connection with the defect that is data communication's more occupation on server system resources and its poor parallel processing ability, in the original on-line automatic-monitoring platform. It improved the shortcomings of the original data communication through the package IOCP model. And we launched an exploratory study about the package IOCP model.
\end{abstract}

Keywords-data communication; optimization; Monitor; Radioactive source

\section{INTRODUCTION}

According to the statistics of 2004, there are 140000 radioactive sources in China now. Among which 110000 radioactive sources are used in the related source units, and 30000 radioactive sources have been stored, the using number of radioactive sources is increasing at a rate of $20 \%$ one year averagely ${ }^{[1]}$. Radiation source is the inevitable outcome of the application of nuclear techniques and an important component of safety management. It will cause a loss of society's economy as well as people's health because of the lose or stolen of radiation source. At the same time, the nuclear threat and the malicious nuclear terrorism is currently an extremely important topic ${ }^{[2]}$. In order to control the radiation sources effectively and store or handle the radioactive waste and abandoned radioactive sources safely, we must establish a set of safe supervision and protection system of nuclear and radiation. ${ }^{[3]}$.

How to make normal operation of a huge supervision protection system, and achieve an effective safe supervision about the nuclear equipment has become an urgent issue of safety environmental protection department to tackle as well as the related nuclear units. Under this case, on-line automatic-monitoring platform of radiation source emerged. On-line automatic-monitoring platform of the radiation sources can achieve three-in-one monitoring on the radiation source and its related facilities. That is: Monitoring the dose rate of the environmental radiation in real time automatically, tracking the position of radiation sources, as well as monitoring the environment of radiation source and so on, so as to make the management department control the dynamic information of the radiation source in time. In addition, it can also deploy emergency monitoring equipment when the nuclear leakage occurs. On one hand, it can be used as a picket fence to alarm the human and animal that enters the alert area; on the other hand, it may throw emergency system into high doses of radiation area which beyond human body can withstand through mechanical facilities. It can provide accurate dose reading reference for emergency command.

With the development of our country's environmental protection, great progress has been made in monitoring technology of radiation source in China. But monitoring network structure of the radiation source is still at the exploring stage ${ }^{[4]}$, especially the data server $R$ \& $D$ predominately stays in the scientific research level, and it has not too much theory for reference, in addition, most research forming PC is developed specifically to the particular needs, so the function is singleness, flexibility and deficient. Although there are some application examples of information communication about I/O completion port at home and abroad, the monitoring data of radiation source is transmitted through the serial ports or wired connection currently, thus the research based on wireless data transmission network is very difficult, especially for its optimization. This article attempts to study the data communication in optimization aspects and hopes to provide some reference for the later.

\section{THE CONTENT OF IOCP}

IOCP is named as the "I/O completion port". IOCP is a mechanism of $\mathrm{I} / \mathrm{O}$ provided by Microsoft, using for efficiently processing equipment in Windows. Equipment can be a file, named pipes, serial, parallel and so on. IOCP provides an efficient core processing mechanism. It manages Winsock communication using the mechanism through specified numerous threads and handles overlapping I/O operation $^{[5]}$.

TABLE I. The Traditional Mode of Socket

\begin{tabular}{|c|c|c|l|}
\hline \multicolumn{1}{|c|}{ Parameter } & CPU & $\begin{array}{c}\text { Me } \\
\text { mor } \\
\mathbf{y}\end{array}$ & \multicolumn{1}{|c|}{ System Status } \\
\hline 100 & $0.3 \%$ & $\begin{array}{c}21 \\
\text { MB }\end{array}$ & Normal run \\
\hline 500 & $20 \%$ & $\begin{array}{c}200 \\
\text { MB }\end{array}$ & $\begin{array}{l}\text { Resource consumption } \\
\text { significantly, occasionally } \\
\text { breaking hery hard, }\end{array}$ \\
\hline 1000 & $50 \%$ & $\begin{array}{l}310 \\
\text { MB }\end{array}$ & $\begin{array}{l}\text { Run } \\
\text { disconnection happens }\end{array}$ \\
\hline
\end{tabular}




\begin{tabular}{|c|c|c|c|}
\hline $\begin{array}{c}\text { Parameter } \\
\text { Clients }\end{array}$ & CPU & $\begin{array}{c}\text { Me } \\
\text { mor } \\
\text { y }\end{array}$ & \multicolumn{1}{|c|}{ System Status } \\
\hline 2000 & $100 \%$ & $\begin{array}{c}480 \\
\text { MB }\end{array}$ & $\begin{array}{l}\text { Unable to operate normally, } \\
\text { system is crashed }\end{array}$ \\
\hline
\end{tabular}

The following is two kinds of communication for pressure test (200 packets are sent per-second) by using traditional Socket and I/O completion port respectively in equal environment. The test results are as shown in Table 1 and table 2. In addition, according to the result of the test, two ways of working performance curves were drawn up, as show in figure 1 . That can come to conclusion that communication mode which adopted IOCP is more suitable for the large-scale data access environment.

TABLE II. WORKING MODE OF THE IOCP

\begin{tabular}{|c|c|c|l|}
\hline \multicolumn{1}{|c|}{ Parameter } & CPU & Memory & \multicolumn{1}{c|}{ System Status } \\
\hline 100 & $1 \%$ & $16 \mathrm{MB}$ & Normal run \\
\hline 500 & $15 \%$ & $110 \mathrm{MB}$ & Normal run \\
\hline 1000 & $20 \%$ & $130 \mathrm{MB}$ & Normal run \\
\hline 2000 & $25 \%$ & $150 \mathrm{MB}$ & $\begin{array}{l}\text { System used resource } \\
\text { is increasing slightly }\end{array}$ \\
\hline
\end{tabular}

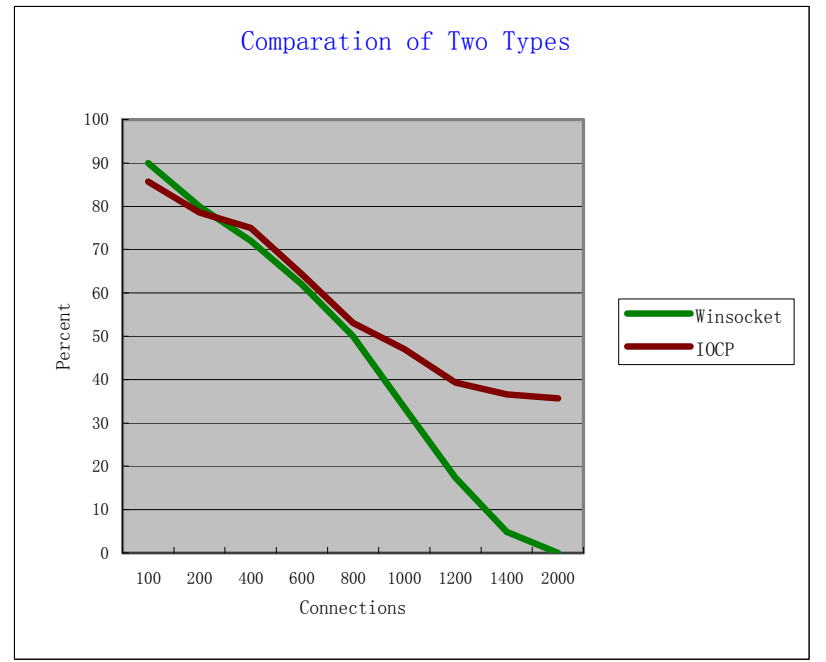

Figure 1. Comparison chart of two kinds of working performance

\section{IOCP REALIZE DATA COMMUNICATION}

As a the data server in the automatic-monitoring platform of the radiation sources, which connected to monitoring terminal and front-end monitoring system, on one hand, it is responsible for the rapid response requests that data processing requests and instructions requests sending from different locations, thousands of the monitoring terminal of the radiation sources. On the other hand, it is responsible for feedback to the front-end control system timely. The working operational state of each terminal reacts to the frontend control system. In addition, it is the underlying data manager of the entire platform. Therefore, the data server plays a pivotal role in the platform-running. Chart of the construction content about the platform shows as in Figure 2.

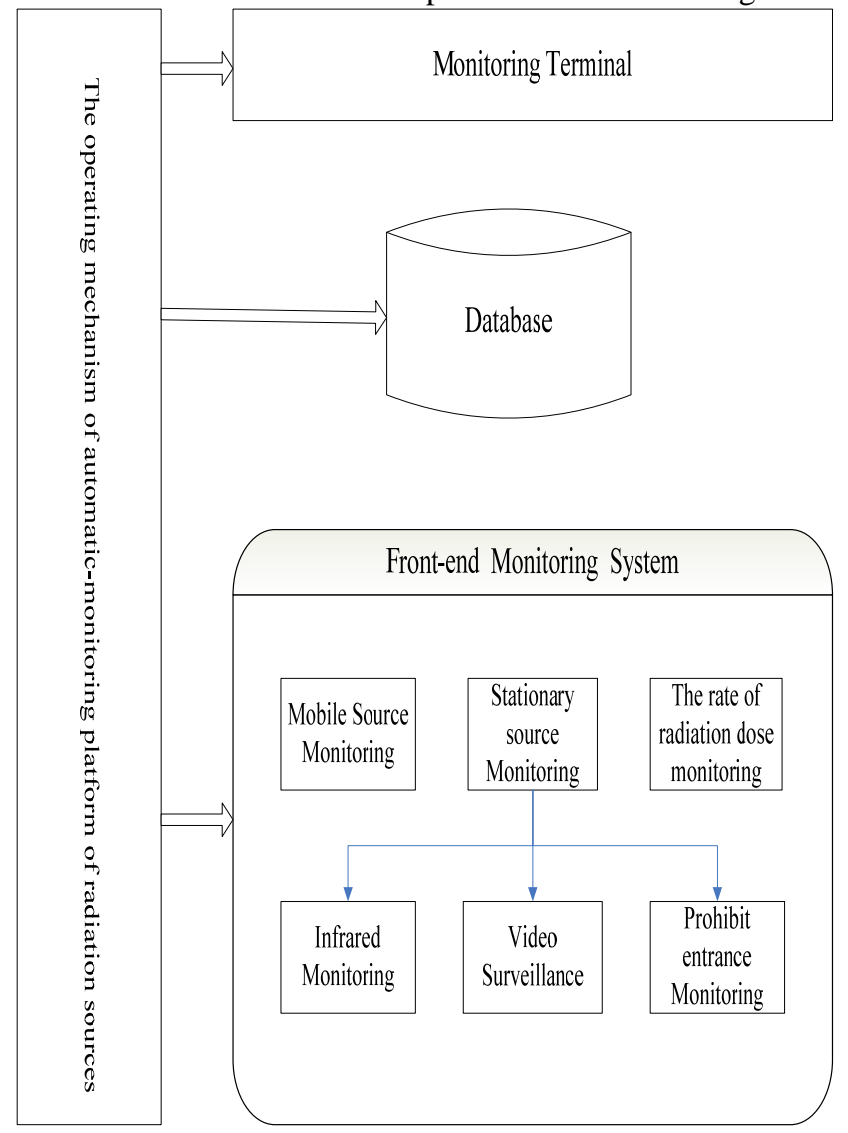

Figure 2. Chart Of The Construction Content About The Platform

Data communication set up a bridge of communication about front-end control system and several-mining terminal. The lack of data communication, the front-end control system was not able to monitor effectively for the monitoring terminal. Monitoring terminal could not upload monitoring data which collected real-time to the monitoring center too. So the entire monitoring-platform lost significance of the running. How to set up a data server and data communication of the monitoring terminal, as well as the studying on optimization of the data communication, it plays a key role, that ensured the efficient operation of a data server and stable operation of the platform.

We discover that in the process of study about on-line automatic-monitoring platform of the radiation sources, transmitting data of wireless network in traditional communication mode is easy to create the network transmission interrupted and form dead link ${ }^{[6]}$, because interference signal of the wireless network is large and signal is instability. In addition, many monitoring terminal's simultaneous access is a severe test for the working ability of data server, if the data server can't response in time as the 
quantity of visit application is too high, the terminal will always be occurred the state of waiting from time to time, and it is very easy to plug the network .The existence of these problems will eventually lead to lower work efficiency of the data server as well as depleted and collapsed resources, at last, The whole platform can not operate normally.

General communication modules create an accept thread firstly, waiting for a user makes a request. And the new thread is created for each user requesting. Then accept thread is suspended, waiting for the arrival of the next user requesting. But the communication modules have problems. The bottleneck of the problem which the application programming create a thread for each user requesting, and these threads will not be suspended, operating systems spend a lot of time to switch the threads. This wastes CPU resources enormously ${ }^{[7]}$.

In view of the above problems and the optimization research about data communication in the monitoring system of traditional radiation source, regarding the data exchange of data resource between data server and the monitoring terminal and the communication working mechanism within data servers main research object, research was done through working model of the I/O completion port in the related fields. These techniques and the technology of the traditional data communication integrate. Compared with the mode of the traditional communication, we use the package of high efficient model of the I/O completion port. This way is more suitable for the data communication of the most visits. The thread is not blocked in the time-consuming I/O operation. And it can be used in the I/O operation service for any users. The threads and the rate of the CPU are increased. Only a few threads can handle the large number of requests of $\mathrm{I} / \mathrm{O}$, avoiding spending much time in scheduling thread for CPU.

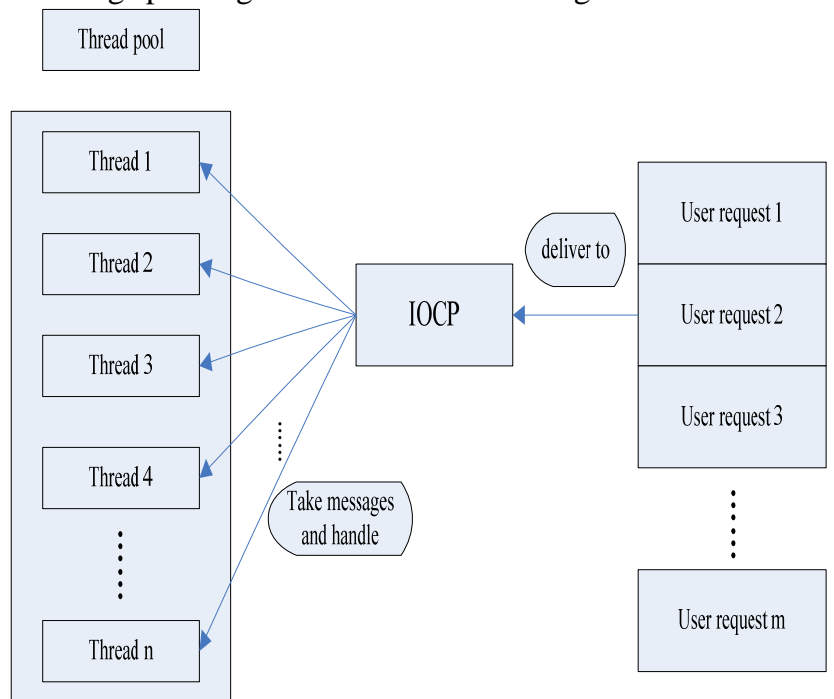

Figure 3. IOCP Model

The situation is that the model of the IOCP (I/O completion port) is set according to the user, and many threads are opened which are used to store in advance in the thread pool. Then the requests of all users are delivered to one I/O completion port, and all threads acquire the user message from the I/O completion port one by one, and then handle them. This will avoid opening a thread for each user. It can not only reduce the resources of the threads, but also improve the utilization rate of the thread. And eventually it realizes communication optimization of the data. As shown in figure 3.

\section{Optimization On The Packets Sort Problem}

The IOCP communication services program using multiple threads to achieve high performance and high concurrency, asynchronous $\mathrm{I} / \mathrm{O}$ request threads and asynchronous processing $\mathrm{I} / \mathrm{O}$ completion threads may not be the same thread, completing order of the worker threads are uncertain. In other words, the former completion of the asynchronous I/O possibly dealt with later, the later completion of the asynchronous I/O may be dealt with firstly. When the user requesting delivered to the IOCP for sending the data, the data that waiting for sending is also re-sorted. According to the problems, it handled memory in accordance with the sequence number, through increasing read and wrote the order numbers in the buffer class. If they had the same sequence number, the data can be processed. Otherwise, the packets of the incorrect sequence number would be cache. It could be processed until the former packet had completed the processing.

\section{CONCLUSION}

This thesis aims to study how to improve the traditional means of communication, in order to adapt to working environment of the radiation sources in on-line automaticmonitoring platform. It exploits advantages of $\mathrm{I} / \mathrm{O}$ completion port fundamentally on handling numerous current accesses, and enhances the data server throughput and efficiency.

\section{REFERENCES}

[1] Qingfu Pu, Xiaomao Zhang. "How to improve the management of radiation source from the accident of radiation". Radiation hygiene of China, vol.2, 2006.

[2] Dong Fang. "Ensure radiation safety on-line monitoring and management system". Monitoring and warning of environment, vol.3, 2011, pp. 26-29.

[3] Lei Yu, Heng Kuang. Construction of remote automatic surveillance management system on radioactive sources [J].Environmental Monitoring and Forewarning. February 2011, Vol. 3, No.1: pp30-32.

[4] Hua Liu, Shunping Zhao, Meiyan Liang, Haijiang Shao, Shiying Ni. "Review and prospect about environment of the radiation in China". Radiation protection, vol.6, 2008, pp.375-376.

[5] Jinghua Zhang, Yuming Zhang. "The research of the IOCP and application in large-scale networks of the communication systems". Computer and modernization,vol.9,2004 ,pp.41-46.

[6] Heping Chen, Jingning Zhou . "The mechanism of the IOCP and implement method of network proxy server”. Computer application, vol.4, 2003.

[7] Yongming $\mathrm{Wu}$, Di He. Design of bottom-module server based on IOCP [J]. Information Technology. 2007(3). 